\title{
Main Lectures
}

ML-01.03

TIME-RESOLVED PROTEIN CRYSTALLOGRAPHY

Keith Moffat*, Ying Chen, Kingman $\mathrm{Ng}$ and Zhong Ren (The University of Chicago, Chicago, Illinois, U.S.A.); Gloria Borgstahl, Duncan McRee, and Elizabeth D. Getzoff (The Scripps Research Institutc, La Jolla, California, U.S.A.)

A complete understanding of mechanism at the molecular level demands knowledge, not just of long-lived, readily observable structures, but also of short-lived intermediates in processes such as catalysis, ligand binding and release, protein unfolding, and phototransduction. Can $x$-ray crystallography, long regarded as a static technique, in fact encompass dynamic processes? A time-resolved crystallographic experiment (Moffat, K. (1989) Ann. Rev. Biophys. Biophys. Chem 18, 309-332.; Time-Resolved Macromolecular Crystallography (Ed. D.W.J. Cruickshank, J.R. Helliwell and L.N. Johnson), Oxford University Press (1992)) has five main components: the $x$-ray source; reaction initiation; reaction monitoring by optical or other techniques; $x$-ray data acquisition in real time; and data reduction and analysis. These are illustrated by our recent work on photoactive yellow protein, on protein unfolding, and on the acquisition and analysis of crystallographic data using 120 ps exposures (Szebenyi, D.M.E., Bilderback, D.H., LeGrand, A., Moffat, K., Schildkamp, W., Smith Temple, B. and Teng, T.Y. (1992) J. Appl. Cryst. 25, 414423).

\section{ML-02.01 DIRECT METHODS IN SUPERSPACE By H. F. Fan, Institute of Physics, Chinese Academy of Scicnces, Beijing 100080, P. R. China.}

With conventional direct methods and many other methods for single-crystal structure analysis, the sample is treated as an ideal crystal, which possesses exact three-dimensional periodicity. However an important category of crystals, incommensurate crystals, do not have three-dimensional periodicity. According to the representation of de Wolff [Acta Cryst. A30 (1974) 777-785] and Janner \& Janssen [Acta Cryst, A36 (1980) 399-408; 408-415], incommensurate crystals are regarded as the three-dimensional hypersection of a higher-dimensional periodic structure. Based on this representation a multi-dimensional direct-method has been developed. With this method incommensurate crystals are first treated in multi-dimensional space to solve the phase problem and then by cutting the resultant multi-dimensional Fourier map with the three-dimensional physical space to obtain the structure. The phasing procedure is divided in two stages. In the first stage phases of main reflections are developed using a modified Sayre equation involving only main reflections. While in the second stage phases of the satellite reflections are derived based on another modified Sayre equation, which relates phases of satellites with those of main reflections. In contrast to other methods of solving incommensurate structures, no preliminary assumption on either the corresponding basic structure or the modulation form is needed through out the process. The method has been 'successfully applied to $\mathrm{X}$-ray as well as electron diffraction data and has been incorporated with an image processing technique in high resolution electron microscopy. Examples are given on the study of incommensurate modulation in the Bi-based high Tc superconductors.
ML-03.01 CONTRIBUTION OF CRYSTALLOGRAPHY TO THE ELUCIDATION OF HYDROLYTIC ENZYMIE MECHANisms. Michael N.G. James, Medical Research Council of Canada Group in Protein Structure and Function, Department of Biochemistry, University of Alberta, Edmonton, Alberta, Canada T6C $2 \mathrm{H} 7$.

Biopolymers such as proteins, nucleic acids and polysaccharides are stable chemical entities that are resistant to hydrolytic cleavage into their constituent building blocks. However, the dictates of various cellular metabolic processes requires the breakdown of the biopolymers once they have served their use. Enzymes such as proteinases, nucleases and glycosidases have evolved to catalyze the degradation of those macromolecules. The hydrolytic cleavage of proteins and peptides is carried out by proteolytic enzymes. They have been categorized into four separate classes, namely the serine, aspartic, metalloand cysteine proteinases. There is a wealth of structural information for various members of each of these enzyme families. Hydrolytic mechanisms have been deduced from the vast biochemical and kinetic literature coupled closely with the stuctural data provided by high-resolution X-ray crystallographic studies of the uncomplexed native enzymes as well as those of enzyme-inhibitor or enzyme-transition state analogue complexes. Both the serine and cysteine proteinases have covalently attached acyl-enzyme intermediates on their reaction pathways whereas the aspartic and metalloproteinases catalyze the hydrolysis without involving covalently attached intermediates. Hydrolysis of peptide bonds involves an attack by an appropriate nucleophile on the carbonyl-carbon atom of the substrate with the resultant formation of a transitory tetrahedral intermediate. Generation of the attacking nucleophile from water or an aminoacid side chain is assisted by a general base which subsequently transfers the abstracted proton to the nitrogen of the leaving group. Electrophilic assistance for the nucleophilic attack is also accompanied by the stabilization of the developing negative charge on the carbonyl-oxygen atom of the tetrahedral intermediate. The detailed stereochemistry of the formation and breakdown of the tetrahedral transition state of the hydrolytic reaction is established. Comparison of the structures of the enzymes in the four proteinase classes shows that the serine, aspartic and metalloproteinases have converged on analogous hydrolytic mechanisms in which nucleophilic attack of the peptide carbonyl-carbon atom is on the re face. In contrast, the cysteine proteinases of known structure likely have the thiolate attack on the si face of the peptide bond. For the proteinases, only serine residues, cysteine residues and water molecules are used to generate the attacking nucleophiles. Imidazole rings and carboxylate groups are used in the role of general base. Electrophilic assistance to hydrolysis is provided by a wide variety of functional groups; even metal cofactors (e.g., $\mathrm{Zn}^{++}$) have been recruited to this role. (Research supported by the MRC Canada.) 